\title{
Defect-mediated curvature and twisting in polymer crystals
}

\author{
Christian Kübel, ${ }^{1}$ Lebzylisbeth González-Ronda, ${ }^{2}$ Lawrence F. Drummy ${ }^{1}$ and David C. Martin ${ }^{1}$ * \\ ${ }^{1}$ Department of Materials Science and Engineering and the Macromolecular Science and Engineering Center, University of Michigan, Ann \\ Arbor, Michigan 48109-2136. USA \\ 2Ford Research Laboratory, P.O. Box 2053-MD 3182-SRL, Dearborn, Michigan 48121-2053, USA
}

Received 15 May 2000; revised 9 August 2000; accepted 10 August 2000

\begin{abstract}
Crystalline polymer solids almost inevitably exhibit defects due to chain ends, chain folding and the limited molecular mobility. The defects result in local (dislocations, grain boundaries) or global (bending, twisting) distortions of the molecular symmetry with pronounced implications on materials properties. Depending on the localization of the deformation, continuous molecular distortions or chain scission are expected, resulting in distinct differences for the mechanical (crack formation) and optoelectronic properties (charge transport and delocalization), which become especially important in the light of the recent extraordinary developments in molecular electronics. Further studies of defect structure and properties in polymers are expected to result in an increasingly sophisticated understanding of the microstructure and microstructural evolution during processing necessary to control and optimize the nano- and micrometerscale structure of organic materials. Copyright (C) 2000 John Wiley \& Sons, Ltd.
\end{abstract}

KEYWORDS: defect; curvature; twist; high-resolution electron microscopy; molecular modeling

\section{INTRODUCTION}

The increasingly demanding applications of polymeric materials require an ever more sophisticated understanding of the relationship between chemical structure, molecular organization and material properties. Defects or local variations in structure are expected to play an important role in limiting macroscopic properties. The impact of structural defects on the optoelectonic and mechanical properties of inorganic materials is fairly well established. ${ }^{1-4}$ Similar ideas have been hypothesized for organic polymers, ${ }^{5,6}$ but a more detailed insight into the role of defects in crystalline polymer and organic molecular solids has only begun to be developed. ${ }^{7-9}$

Characteristic deformation modes which have been observed at high resolution in polymer crystals include bending and twisting (Fig. 1). ${ }^{10-17}$ Such deformations disrupt the three-dimensional periodicity of the crystal, and are thus of fundamental scientific interest and have important practical implications. For example, it has been argued that for semiconducting oligothiophenes, which

*Correspondence to: D. C. Martin, Department of Materials Science and Engineering, University of Michigan, Ann Arbor, Michigan 48109-2136, USA.

E-mail: milty@umich.edu

Contract/grant sponsor: Alexander-von-Humboldt Foundation.

Contract/grant sponsor: Rackham Graduate School.

Contract/grant sponsor: NSF IGERT Center on Molecularly Designed

Electronic, Photonic, and Nanostructured Materials.

Contract/grant sponsor: National Science Foundation; Contract/grant number: DMR-9707974, DMR-9704175, and DMR-0084304. are of interest for organic field-effect transistors, local curvature allows the contact and continuity of molecular interactions necessary to obtain high carrier transport efficiencies. ${ }^{10}$ However, systematic efforts to quantify the nature and extent of the curvature have yet to be made. There is also a need to identify the specific dislocations that have been hypothesized to accommodate these distortions. Much more work is needed to classify the mechanisms of this response and to elucidate the localization of the deformation. This information should prove useful in designing processing schemes to a)

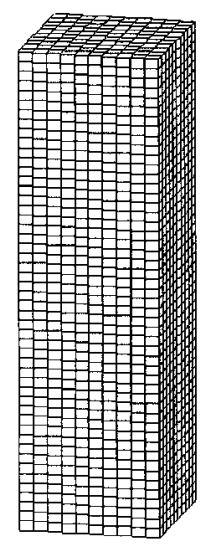

b)

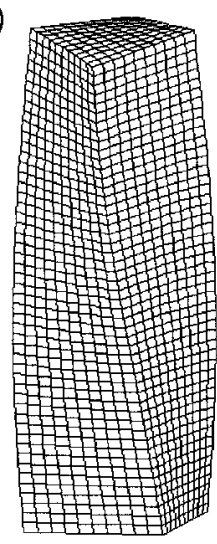

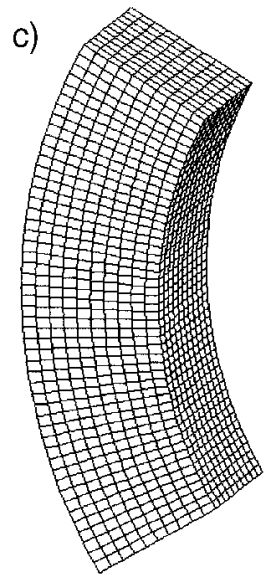

c)
Figure 1. (a) Perfect crystal with periodic three-dimensional order. (b) Coherently double-twisted crystal geometry due to twisting around its long axis relaxing the strain by twist perpendicular to the long axis. (c) Coherently bent crystal 
control and optimize the development of these distorted microstructures.

In this paper, work is reviewed that has identified the structure and impact of specific defects on macroscopic properties in organic and polymer materials. We then focus on the characteristic distortions of curvature and twisting of polymer and organic crystals and the defects that mediate these deformations. Furthermore, molecular simulations are discussed, providing new insights about defect structures in crystalline polymers and organic solids.

\section{DEFECTS IN POLYMERS}

To maximize performance in a variety of applications, it is often desirable to order materials to promote a preferred molecular alignment and facilitate interactions between molecules. However, this organization will inevitably result in the presence of defects disrupting the symmetry, which will lead to limitations in ultimate performance. Fundamental studies of defects therefore make it possible to learn precisely how it is that they control behavior, which can then lead to more rational control of structure-property relationships. It is also possible that devices could be designed such that the properties of defects themselves could be exploited to technical advantage, as has been proposed for inorganic semiconductors. ${ }^{18}$

In single-crystal inorganic semiconductors, it is well known that the presence of structural defects limits the macroscopic properties of electronic and optoelectronic devices. ${ }^{1-3,19,20}$ It has likewise been hypothesized that the presence of structural defects such as grain boundaries should limit the macroscopic properties of electrically active molecular crystals. ${ }^{21-23}$ Semiconducting organic and polymer materials are of considerable interest for a variety of optoelectronic applications. ${ }^{10,24,25}$ For example, pentacene and substituted oligothiophenes are under commercial development as the channel layer in thinfilm field-effect transistors. ${ }^{26-29}$ Organic semiconductors have now demonstrated charge carrier mobilities of $3 \mathrm{~cm}^{2} \mathrm{~V}^{-1} \mathrm{~s}^{-1}$, ${ }^{29}$ which is similar to that of hydrogenated amorphous silicon. Organic materials are attractive because of their ability to be rapidly solution-processed near room temperature, which should make it possible to use inexpensive, large-scale printing techniques for device manufacture. ${ }^{30}$ It is also thought that they will be much more flexible and pliable than the relatively brittle single-crystal silicon and gallium arsenide substrates used in traditional inorganic semiconductors.

However, a detailed understanding of structureproperty relationships in organic crystals remains incomplete. The scientific understanding of dislocation structure, energetics and mobility is not nearly as well developed for molecular and polymer crystals as it is for metallic and inorganic crystals. This seems to be largely due to the limited number of high-resolution studies of the local microstructural organization near defects and the correlation of this information with macroscopic electrical and mechanical behavior. However, a few studies have provided a theoretical framework for understanding the expected behavior and impact of dislocations in polymer crystals. The possibility of dislocation-mediated plasticity in polymers was discussed by Frank et al. ${ }^{5}$ Zaukelies described dislocationmediated mechanisms of deformation in oriented nylons. ${ }^{31}$ Keith and Passaglia discussed the geometry of dislocations, particularly with respect to the chain-folded surfaces of the crystallites, and in terms of the directions of easy slip. ${ }^{6}$ Holland and Lindenmeyer obtained evidence for screw dislocations between chain-folded lamellae, and observed the dissociation of the core into partials. ${ }^{32}$ Predecki and Statton considered the nature and extent of the disorder that would be introduced by incorporating chain ends into a polymer crystallite. ${ }^{33}$ Shadrake and Guiu have discussed the line energies and geometries of dislocations in polyethylene. ${ }^{34}$

It has been argued that dislocations with Burgers vectors $b=[001]$ control the plastic deformation of aliphatic semicrystalline polymers near room temperature. ${ }^{35-38}$ Most recently, it has been shown that the yield strength of polyethylene crystals as a function of crystal size and temperature can be rationalized by screw dislocation-mediated slip. ${ }^{39}$ To obtain the best fit of the data with theory, it was necessary to assume a model that accounted for the possibility that the dislocation core energy was a function of temperature.

Erickson proposed a defect mediated-process to explain the irreversible elongation of highly oriented polymer fibers. ${ }^{40}$ It was argued that the annihilation of defects during creep could explain the increasing modulus and the total elongation during the process. ${ }^{41}$ Schuppert explained the time dependence of creep in fibers by assuming the transport of defects towards chain ends in combination with annihilation of defects in neighboring chains. ${ }^{42}$

In order to perform systematic studies of defects, our research group has been developing schemes to introduce specific crystallographic defects into ordered, optoelectronically active organic molecular and polymer crystals. This work has involved systematic studies of the carbazolyl-substituted poly(diacetylene) (DCHD), which can be grown into macroscopically large single crystals from the precursor monomer. ${ }^{43}$ The initial approach focused on grain boundaries as the model defect. Preparation of polymer bicrystals with a single grain boundary with controlled geometry made it possible to study the properties of these engineered defects, which limit charge carrier transport and mechanical stress transfer across the interface (Fig. 2). By examining the change of macroscopic properties of the bicrystals with increasing grain boundary angle, specific information about the defect sensitivity of crystalline organic 
a)

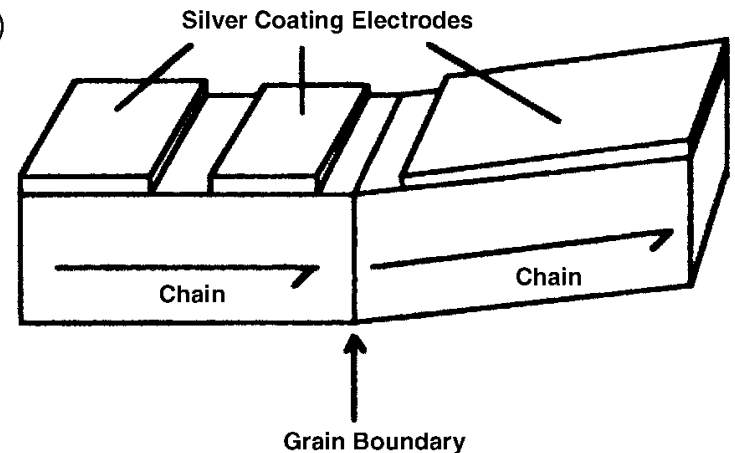

b)

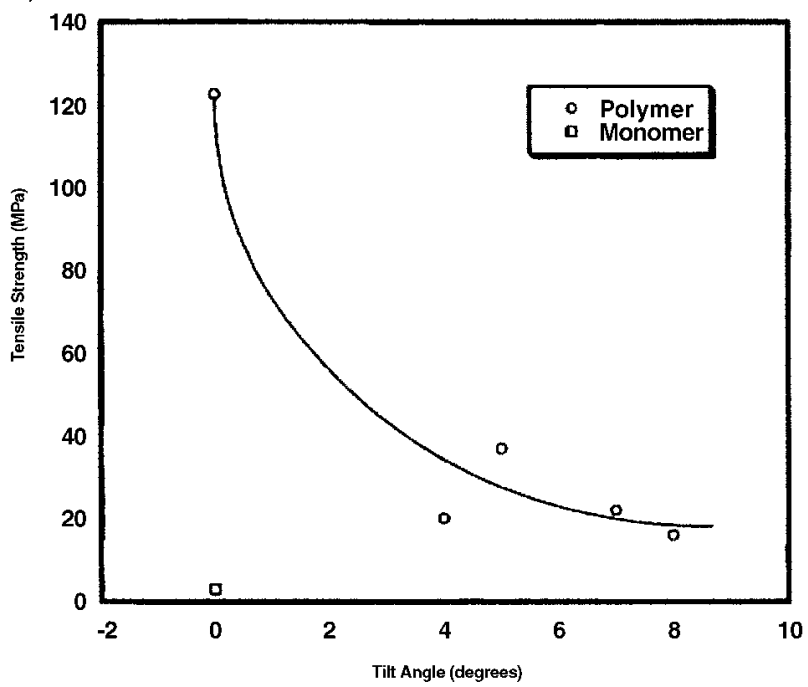

c)

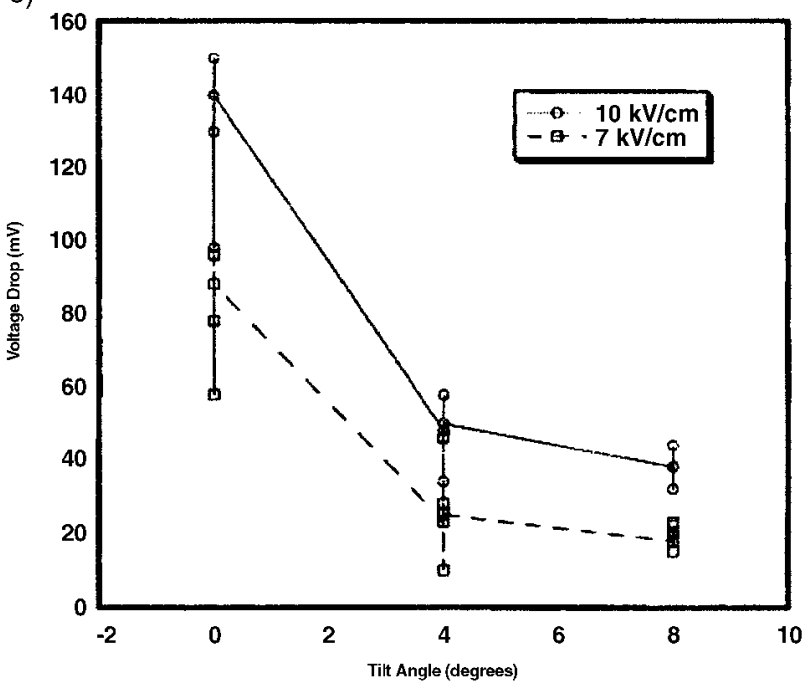

Figure 2. (a) Schematic diagram of a polyDCHD bicrystal. (b) Plot of the tensile strength of polyDCHD bicrystals decreasing with increasing tilt angle. The tensile strength of the polymer bicrystals is still significantly higher than that of a monomer single crystal. (c) Maximum transient voltage drop across a 50 $\Omega$ resistor as a function of grain boundary tilt angle for two different applied fields: 7 and $10 \mathrm{kV} \mathrm{cm}^{-1}$. Reprinted from Liao J, Martin DC, 'Construction and Characterization of Grain Boundaries in Polymer Bicrystals,' Macromolecules, 1996; 29: 568, with permission of the American Chemical Society polymers has been obtained. ${ }^{44}$ This has confirmed that the electronic transport and mechanical integrity of grain boundaries decrease rapidly for small deviations $\left(<15^{\circ}\right)$ away from zero misorientation (Fig. 2). Details about the grain boundary microstructure have been revealed by low-dose high-resolution electron microscopy (HREM). Reliable low-dose HREM experiments require considerable care in controlling beam damage and in comparing the images obtained with the theoretically expected contrast, given information about the molecular packing within the unit cell. ${ }^{10,45-51}$

In HREM imaging, optimum phase contrast for crystal planes of spacing $d$ is obtained at a certain value of objective lens focus which depends upon the contrast transfer function of the microscope. ${ }^{46}$ When $d$ is reasonably large $(>0.6 \mathrm{~nm})$, the optimal focus $\Delta f$ is given by $\Delta f=d^{2} / 2 \lambda$, where $\lambda$ is the wavelength of the electrons (e.g. $\lambda=1.64 \mathrm{pm}$ at $400 \mathrm{kV}$ ). Optimum resolution for planes of a minimum spacing $d_{\mathrm{m}}=0.66\left(C_{\mathrm{s}} \lambda^{3}\right)^{1 / 4}$, where $C_{\mathrm{s}}$ is the spherical aberration coefficient of the objective lens, occurs at extended Scherzer defocus, which is given by $\Delta f=-\left(3 / 2 C_{\mathrm{s}} \lambda\right)^{1 / 2}$. Since the lateral and longitudinal $d$ spacings in polymers can be very different, it is a significant advantage to take a series of HREM images under predetermined focusing conditions. We have now confirmed the ability to acquire sequentially such a series of HREM images from the same area of a DCHD nanocrystalline sample. The total dose per image is $\sim 0.05 \mathrm{C} \mathrm{cm}^{-2}$ at magnifications which made it possible to image lattice spacings as small as $0.18 \mathrm{~nm}$ (C. Kübel and D. C. Martin Philos Mag. A submitted).

Other groups are also beginning to extract details about defect-limited macroscopic properties in optoelectronically active organic crystals. Frisbie and co-workers have examined the electrical transport across individual defects in organic sexithiophene crystals by conducting probe atomic force microscopy (CP-AFM) ${ }^{52-54}$ From measurements of $I-V$ curves on thin sexithiophene crystals as a function of position away from a nanofabricated gold electrode, they estimated typical grain resistivities of $\sim 100 \Omega$ and a contact resistance to the gold electrode of $\sim 100 \mathrm{M} \Omega .{ }^{54}$ Across a defect that they identified as a grain boundary, a significant increase in resistance was observed corresponding to a grain boundary resistivity of about $25 \mathrm{G} \Omega .{ }^{53}$ The beauty of the CP-AFM technique is that the electrical properties of individual defects can be directly measured at extremely high resolution. However, the limited resolution of the blunt, metallic-coated AFM tip makes it difficult to image directly the molecular structure and relaxation phenomena near the interface.

It is well established that small-angle grain boundaries in crystalline interfaces can be described in terms of arrays of dislocations, where the nature of the dislocation (edge or screw) depends on the geometry of the misorientation (tilt or twist). ${ }^{55,56}$ As the misorientation $\theta$ increases, the spacing $d_{\mathrm{d}}$ between dislocations of 
a)

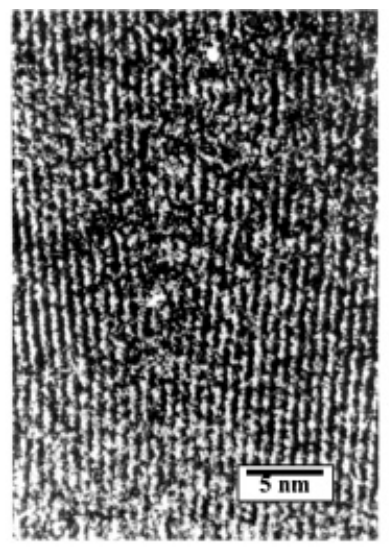

b)

c)
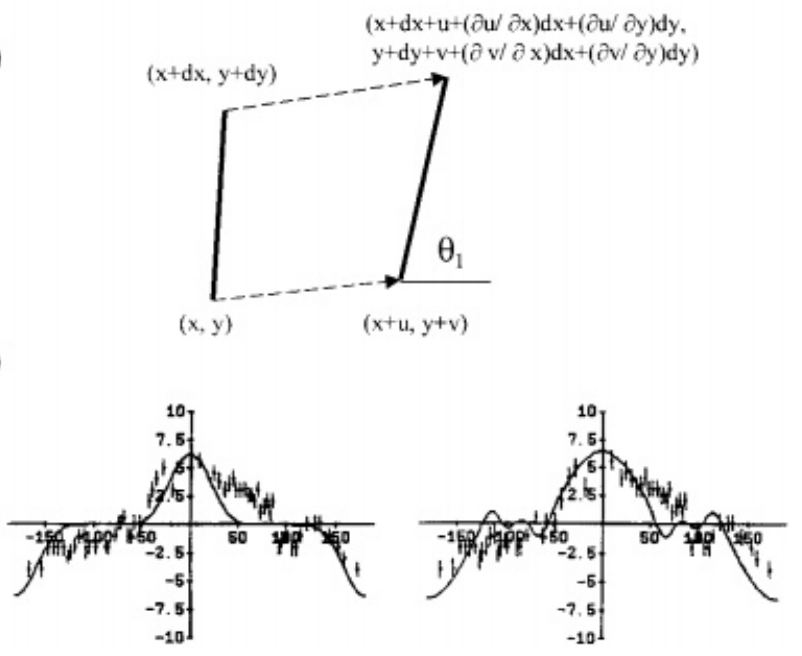

Figure 3. (a) A $b[200]=2.4 \mathrm{~nm}$ three chain-end edge dislocation in polyDCHD. The fringes correspond to the (200) lattice planes. (b) Schematic diagram of the method used to predict tilt $(\theta)$ of any lattice plane given by $\mathrm{d} x$ and $\mathrm{d} y$ around a dislocation whose displacement fields are given by $u$ and $v$ (c) Experimentally observed tilt measured from the HREM image in comparison with predictions using columnar liquid crystal $\left(\lambda_{3}=0.8 \pm 0.1 \mathrm{~nm}\right)$ and anisotropic elastic theory $(W=3.5)$. Reprinted from Wilson PM, Martin DC, 'Quantitative Measurements of Polymer Chain-end Edge Dislocation Strain Fields by High Resolution Electron Microscopy,' Macromolecules, 1996; 29: 842 with permission of the American Chemical Society

Burger's vector $b$ will decrease as.

$$
d_{\mathrm{d}}=b / \sin \theta
$$

The dislocation cores represent regions where the packing is disrupted from the symmetry of the perfect crystal. Polymer chains near the core will therefore be distorted, and may even be broken completely. Grain boundaries can be considered as regions where symmetry and molecular connectivity are disrupted (near the dislocation core), and regions where structural relaxations allow the molecular connections and interactions to remain intact (between dislocations). Hence studies of the dislocations themselves would provide fundamental insight about expected behavior of small-angle grain boundaries. Furthermore, this also shows the importance of studying the nature and extent of strain localization near the core. Dislocations with widely spread cores should have a dramatically different influence on transport properties and plasticity than those in which the deformation is localized.

Wilson and Martin have examined the distortions near the core of chain-end edge dislocations in polydiacetylenes by HREM. ${ }^{57}$ By measuring the tilt angle of the (200) lattice planes as a function of azimuthal angle around the defect, they were able to estimate the anisotropy of the polymer's mechanical properties. Efforts to extend this analysis to more isotropic, microphase separated block copolymers have revealed that a more detailed description of the deformation field is necessary. The previous analysis related tilt of the polymer chain directly to the local shear strain calculated from anisotropic dislocation theory. For a chain-end edge dislocation in DCHD, this proved to be a good approximation. However, dislocations disrupt the translational symmetry of a material by inducing not only shear strains, but also rigid body rotations and local tension and compression. A more general and accurate way of examining the deformation near a dislocation is to use the two-dimensional displacement fields predicted by linear elastic dislocation theory (L. F. Drummy, D. C. Martin and I. G. Voigt-Martin, in preparation). Using this method we can relate the measured tilt of any unit cell plane from HREM dislocation images and compare it with the predicted tilt calculated from the theoretical displacement fields. Derived from isotropic ${ }^{58,59}$ or anisotropic $^{60}$ dislocation theory, these displacement fields can be altered to fit the data best by varying the input elastic constants. It is therefore possible to extract information about elastic constant anisotropy of the material.

For a more accurate analysis and modeling of dislocations, simultaneous imaging of different sets of lattice planes is necessary. ${ }^{61}$ In previous studies of dislocations in molecular systems such as DCHD we did not obtain direct images of the molecular packing along the polymer backbone, for which the $d$ spacings are smaller $\left(d_{001}=0.49 \mathrm{~nm}\right.$ for DCHD). However, we have recently demonstrated that with improved sample preparation techniques, and with access to better instrumentation, we can now reliably obtain images of smaller lattice spacings in DCHD thin films resulting in a more detailed description of the molecular structure (Fig. 4). (C. Kübel and D. C. Martin Philos Mag. A in press).

The energy for a dislocation is nominally proportional to the square of the Burger's vector. ${ }^{62}$ This leads to the relationship known as Frank's rule, which is that a dislocation $b_{1}$ will dissociate into partials $b_{2}$ and $b_{3}$ if the sum of the squares of $b_{2}$ and $b_{3}$ is smaller than the square of $b_{1}$. When the dislocation dissociates, the distance 


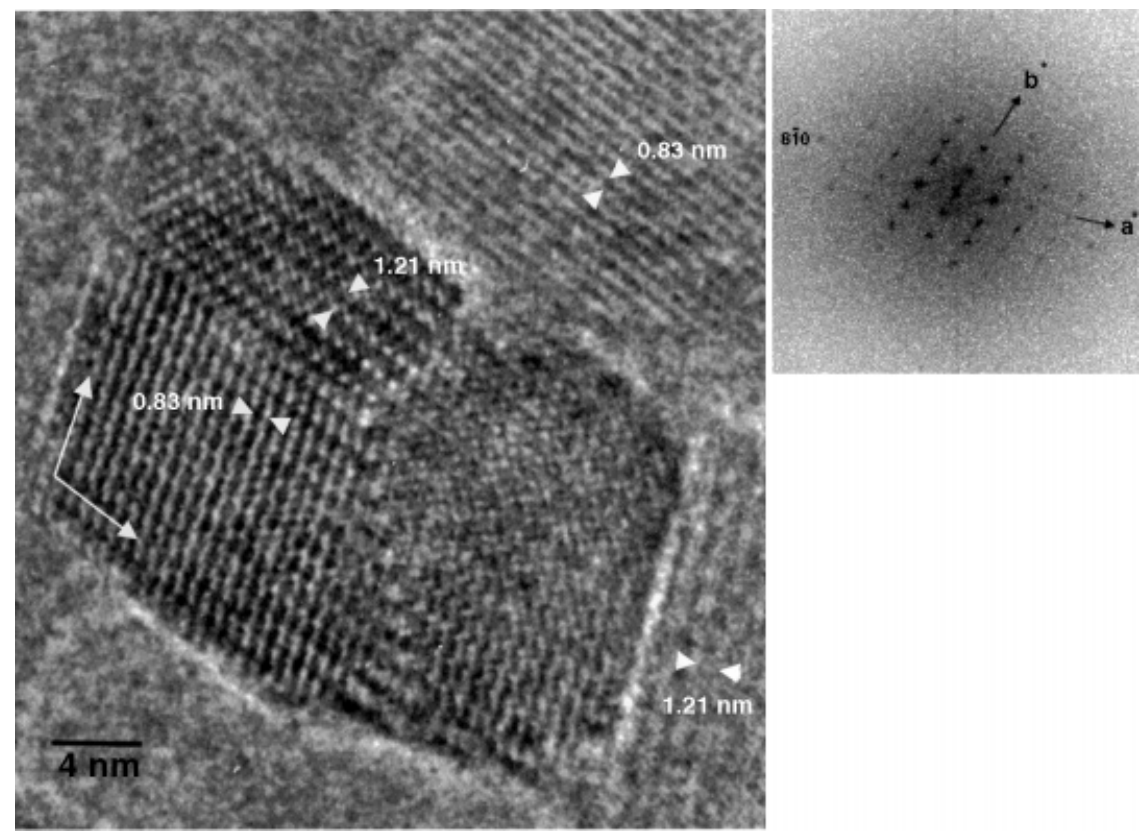

Figure 4. LD-HREM image and fast Fourier transformation (FFT) of a DCHD nanocrystal imaged on the [001] zone revealing three sub-domains separated by a small angle grain boundary.

between the partials is determined by the increase of energy due to the breaking of symmetry along the plane (i.e. the stacking fault energy $\gamma$ ). The expected spacing $d_{\text {s }}$ between the partial dislocations at equilibrium is directly related to the shear strength $G$ and inversely to $\gamma:{ }^{62}$

$$
d_{\mathrm{s}}=G b^{2} / 4 \pi \gamma
$$

For materials with high stacking fault energy, it is expected that the dislocations will not dissociate (small $\left.d_{\mathrm{s}}\right)$. However, for polymers, $\gamma$ should be low on $(h k 0)$ planes because there are only non-bonded interactions between molecules. Therefore, we suspect that dislocations on $(h k 0)$ in polymers will typically tend to break up into partials. For metals, stacking fault energies are typically in the range $20-40 \mathrm{~mJ} \mathrm{~m}^{-2}$, whereas grain boundaries have energies of $300-1000 \mathrm{~mJ} \mathrm{~m}^{-2}$ and crystal vapor energies of $1000-3000 \mathrm{~mJ} \mathrm{~m}^{-2}{ }^{63}$ Although the precise stacking fault energies for polymer crystals are not well known, surface energies for amorphous and semicrystalline polymers are typically in the range 10 $40 \mathrm{~mJ} \mathrm{~m}{ }^{-2} \cdot{ }^{64}$ Measurements of the melting-point of polyethylene as a function of crystal size have led to an estimate of the basal surface free energy for polyethylene of $\sim 80 \mathrm{~mJ} \mathrm{~m}^{-2} \cdot{ }^{65}$ Estimates of the stacking fault energy can in principle also be obtained from HREM images of dislocations in polymer crystals as the separation of the partials into stacking faults can be directly related to the stacking fault energy using Eqn. (2).

Atomistic computer simulations have revealed information about the structure and energetics of dislocations in organic crystals, although the number of studies is still limited. Mokichev and Pakhomov studied the core structure of edge dislocations in naphthalene. ${ }^{66}$ Bacon and Tharmalingam studied the structure of edge dislocations in polyethylene, and obtained an estimate of the Peierls stress, which is the flow stress required to maintain dislocation motion in a solid with periodic, translational order (i.e. a crystal). ${ }^{67}$ Ide et al. studied the core structure of edge and screw dislocations in anthracene and found that the distortions were anisotropically distributed around the line of the defect. ${ }^{68,69}$

A detailed atomistic study of the effect of dislocations in a plastic explosive was published by Kuklja and Kunz, who performed $a b$ initio Hartree-Fock calculations combined with many-body perturbation theory on edge dislocations in crystals of cyclotrimethylene trinitramine (RDX). ${ }^{70}$ The dislocations were shown to produce local electronic states in the optical band gap. Under pressure these electronic states created favorable conditions for $\mathrm{N}-\mathrm{NO}_{2}$ chemical bond rupture, resulting in hot spots for the detonation.

We have conducted molecular mechanics simulations of dislocations in polymer crystals with Burger's vector $b=[001]$ to obtain a better understanding of the molecular distortions induced by the dislocations. To isolate the dislocation, we constructed superlattices that topologically trap the defect into a larger, periodic unit cell. Figure 5(a) shows an energy-minimized configuration for a dislocation with Burger's vector $b=[001]$ and line direction $=[100]$. This defect was created starting with a perfect crystal of the DCHD monomer and polymer, 
a)

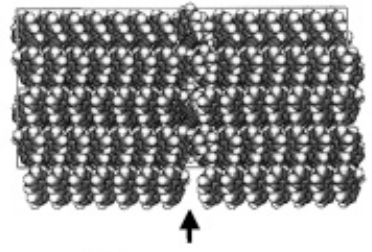

b)

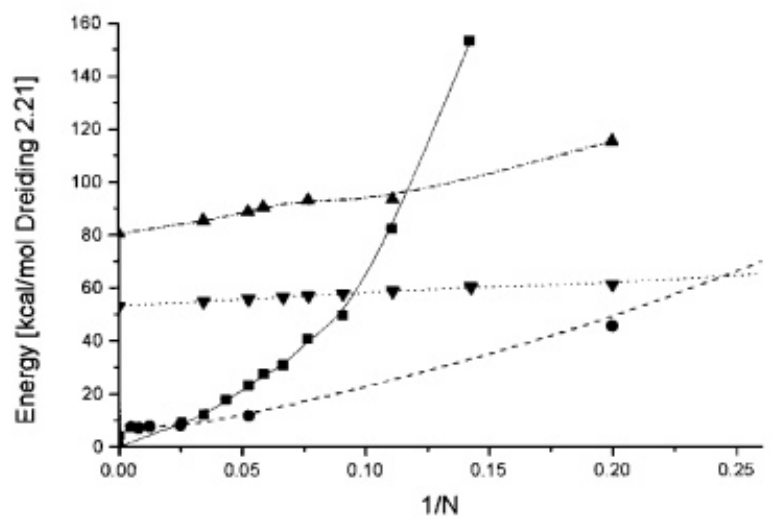

Figure 5. (a) Minimized structure of a topologically trapped [001] dislocation in a DCHD monomer (localized at arrow) and polymer crystal (delocalized over about 7-10 monomer units) $(N=15)$. (b) Plot of the energy per monomer unit for $\operatorname{DCHD}$ monomer $(\boldsymbol{\nabla})$, polymer $(\boldsymbol{\Delta}), \operatorname{MPDI}(\boldsymbol{\square})$ and $\operatorname{PMBT}(\boldsymbol{\bullet})$ vs $1 / N$

respectively. The crystal structure was then expanded twice in the [010] direction and $N$ times in the [001] direction along the molecular axis. The length of one chain was reduced by one monomer unit to $N-1$. The structure was then allowed to minimize, using a combination of energy minimization and NPT molecular dynamics annealing with the Dreiding 2.21 force field, charge equilibration and variable unit cell parameters. The simulations were performed for varying chain length $N$ (typically 3-20) to simulate different defect densities. Figure 5(b) is a plot of the energy per repeat unit vs $1 / N$ with the slope corresponding to the dislocation chemical potential. In case of the DCHD monomer the non-linear displacement field and relatively low energy of the dislocation resulted in a localized deformation and crack formation. As expected, the strong intramolecular bonding of the DCHD polymer results in a higher dislocation energy and the dislocation cores are delocalized over about 7-10 repeat units. For a detailed comparison with experimental HREM images, the defect density perpendicular to the polymer backbone has to be further reduced, but these simulations still make it possible to estimate the defect energy and also the delocalization of the dislocation (Fig. 5). Similar results have been also been obtained for poly(methylbithiazole) (PMBT) and $\operatorname{poly}(m$-phenylene isophthalamide) (MPDI), showing that different intermolecular interactions result in a variation of the defect energy and extent of the localization (D. C. Martin, L. Drummy, L. Gonzalez and C. Kübel, poster presented at the American Physical Society Meeting, Minneapolis, 2000).

\section{CURVATURE IN POLYMER CRYSTALS}

Perfect single crystals are composed of translationally ordered molecules or atoms, which define a local lattice given by three non-parallel basis vectors. Individual dislocations disrupt the local symmetry, but the global translational symmetry is maintained. However, macroscopic curvature is inconsistent with global translational symmetry. Because of the geometric incompatibility between crystallinity, which is trying to promote local translational order, and the external curvature, it is expected that the structure will try to find some intermediate arrangement that satisfies both constraints as well as possible. We therefore anticipate that studies to elucidate the specific mechanisms of bending will provide insight about the relative strengths of these various modes of deformation.

The bending of lattice planes is well known in the turbostratic graphite form of carbon, which forms layered structures with a characteristic spacing of $0.34 \mathrm{~nm}$. Curved carbon sheets consisting of five-, six- and seven-membered rings compose the fundamental units of fullerenes, carbon nanotubes and concentric graphitic onions. ${ }^{71}$ HREM images of these carbon onions have shown variations in the lattice spacing due to the increased pressure at the core of the particle. The pressure inside the particle is sufficient to induce the structural transformation from graphitic carbon to diamond. ${ }^{72}$ Curvature is also seen in other inorganic systems, such as in the layered silicate crysotile. ${ }^{73}$ Because of distortions between tetrahedral sheets of oxide layers, there are curvatures that can roll the lattice up into a structure similar to a carpet roll. Curvature has also been seen in clusters created by inorganic precipitation from a liquid crystalline precursor. The resulting structures have apparent similarities to naturally curved objects such as animal shells. ${ }^{74,75}$

There have now also been many instances where crystals have been designed to exhibit curvature due to external boundary conditions such as deformation gradients, free surfaces or electric fields. Martin and Thomas found direct evidence for local curvature in the interior of kink deformation zones in the rigid-rod polymer poly ( $p$-phenylenebenzobisoxazole) (PBO) using HREM. $^{76}$ Kink formation results in lateral compressive deformation on the inside and lateral tensile deformation on the outside of the kink. Owing to the asymmetric nature of the intermolecular potential, the tensile side exhibits sharp changes in molecular orientation and material density, whereas the compressive side is more diffuse. Jones and Martin observed that kink formation is sensitive to intermolecular interactions resulting in a change of the bending mode from single molecules in PPTA (Kevlar 49) to microfibrillar bending in crosslinked PPXTA as indicated by the increasing width of the kink deformation bands. ${ }^{77}$

The presence of local curvature has also been seen by 
a)

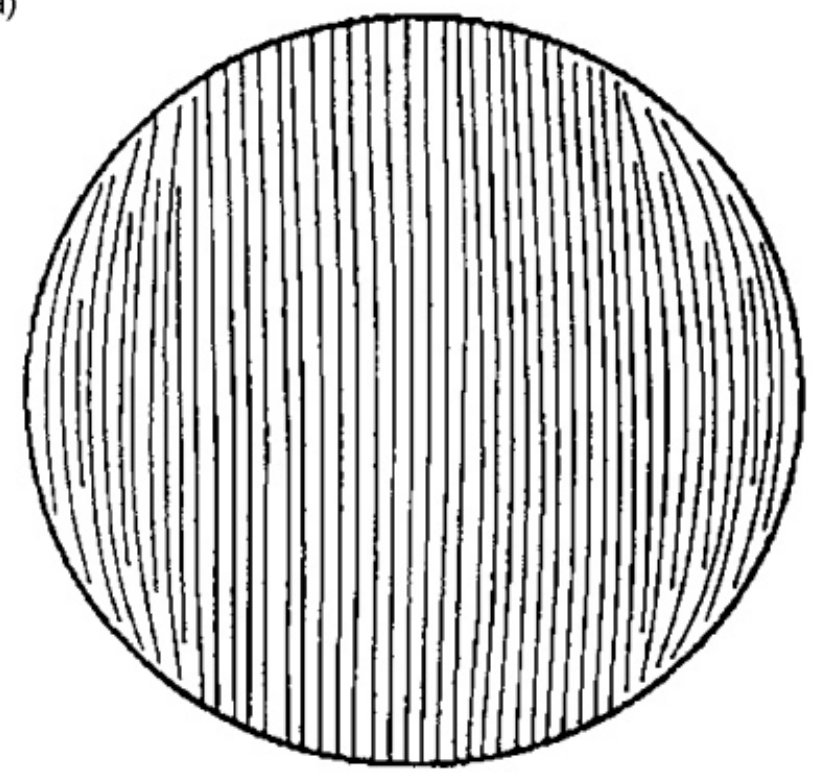

b)

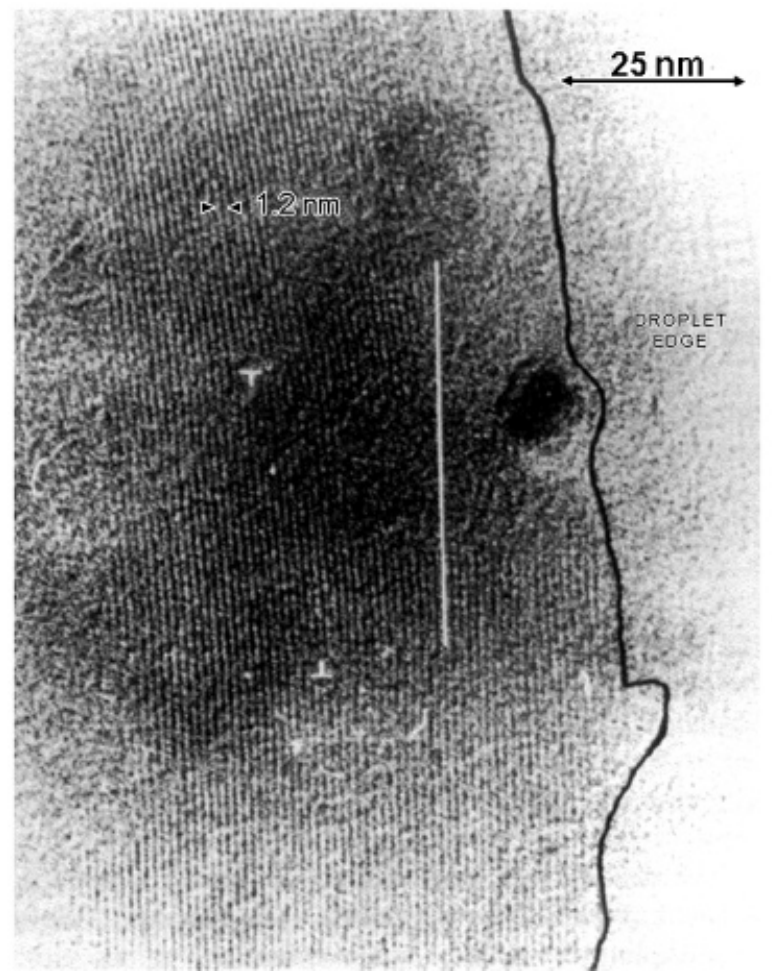

Figure 6. (a) Schematic representation of the polymer backbone in DCHD droplets. (b) LD-HREM image of DCHD near the edge of a droplet exhibiting almost uniform curvature to comply with the surface constraints. Reprinted from Wilson PM, Martin DC, 'Dislocation-Mediated Lattice Bending in DCHD Poly(diacetylene) droplets,' J. Mater. Res. 1992, 7: 3150, with permission from the Materials Research Society

HREM near the surfaces of polyimide droplets. ${ }^{12}$ Wilson and Martin reported the curvature of the carbazolylsubstituted diacetylene DCHD in droplets deposited on surfaces $^{11}$ and formed by evaporation in a droplet

Copyright () 2000 John Wiley \& Sons, Ltd.

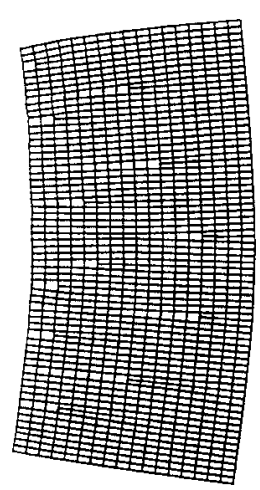

Figure 7. Schematic representation of dislocation mediated lattice curvature (polymer backbone nominally vertical). Reprinted from Wilson PM, Martin DC, 'DislocationMediated Lattice Bending in DCHD Poly(diacetylene) droplets,' J. Mater. Res. 1992, 7: 3150, with permission from the Materials Research Society

tower. ${ }^{78}$ HREM revealed the polymer backbone to be oriented in the plane of the droplet as depicted in Fig. 6(a). The surface constraints induce almost uniform curvature for polymer chains close to the droplet edge [Fig. 6(b)]. It is expected that dislocations with $b=[001]$ accommodate the curvature distortions to minimize strain along the chain axis (Fig. 7).

Radii of curvature of $25-75 \mathrm{~nm}$ were observed in round nanoparticles of DCHD oligomers which were prepared by precipitating a DCHD solution into water followed by fast solid-state UV polymerization of the diacetylene monomers. ${ }^{79}$ Katagi et al. did not obtain

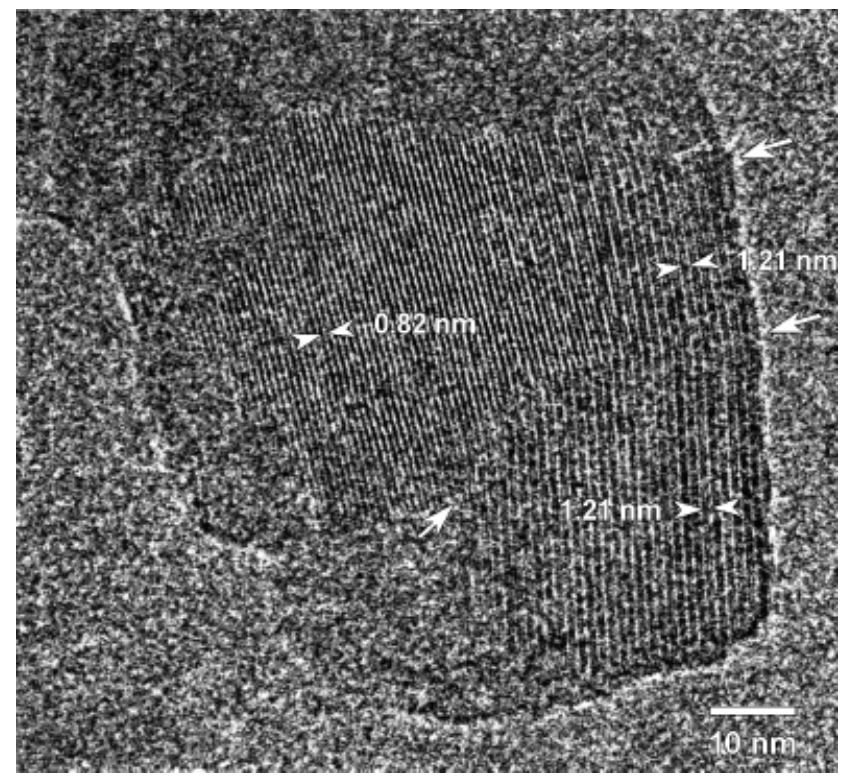

Figure 8. LD-HREM image of lattice fringes in a DCHD nanocrystal exhibiting localized bending (right arrows) and a dislocated grain boundary between two domains (left arrow) 
details of the nanocrystal organization, but it could be assumed that the fast polymerization conditions would result in almost amorphous nanoparticles, which exhibit only a weak resistance towards the distortions induced by surface minimization. In contrast, crystallization for 10 min before UV polymerization results in almost rectangular polymer nanocrystals. ${ }^{79}$ We used LD-HREM to show that the crystal structure of these DCHD nanoparticles is in agreement with the bulk structure (C. Kübel and D. C. Martin Philos. Mag. A in press). The three-dimensional order in the crystalline material gives rise to a much higher resistance towards surface minimization, resulting in lattice fringes that typically exhibit localized bending or kink formation (Fig. 8).

Samori et al. obtained STM images of aggregated 'nanoribbons' of alkyl-substituted poly( $p$-phenyleneethylene) derivatives ${ }^{80,81}$ Either uniformly curved structures or stiff, straight segments connected by local bending were formed after evaporation on to mica substrates, depending on the quality of the solvent. ${ }^{80}$

Lovinger et al. obtained HREM evidence for the local distortions of the $3.52 \mathrm{~nm}$ (200) spacing corresponding to the end-to-end distance of end-functionalized oligothiophenes. ${ }^{10}$ The fringes were observed to be locally parallel to the edges of rod-like crystals that percolated together into an interconnected network. Multi-slice simulations were used to correlate the dark lines in the images with the self-assembled layers of hexathiophene units, through which charge carriers are expected to be transported. Close inspection of the images showed evidence for dislocations and grain boundaries, and also instances of local curvature. However, the detailed mechanisms of presumably dislocation-mediated lattice curvature in this system still remain unclear owing to the absence of HREM images of the smaller molecular spacings.

The relationship between the curvature of crystals and the local dislocation density was originally discussed by Nye. ${ }^{82}$ This analysis showed that the curvature strain was directly related to the state of dislocation of the sample, as defined by the 'dislocation tensor.' One implication was that the curvature strain $\kappa$ could be written as $\kappa=n b$, where $n$ is the dislocation density and $b$ the Burger's vector. Read used these ideas to describe the plastic behavior of bent beams. ${ }^{83}$ Bilby et al. showed that dislocations could describe the twisting deformation of cylindrical beams. ${ }^{84}$ The dislocations could lead to slip on planes either perpendicular ('stack of coins') or parallel ('twisting pencils') to the long axis of the cylinder.

Srolovitz et al. developed a theory to describe the variations in energy expected for different modes of introducing curvature in thin films. ${ }^{85}$ They discussed the limiting cases of (1) coherent bending of an entire assembly and (2) incoherent bending of easily sliding layers of some molecular thickness $a$. They showed that the bending of a film of thickness $h$ and $a$ curvature $\kappa$ (corresponding to a radius of curvature $r=1 / \kappa$ ) has an

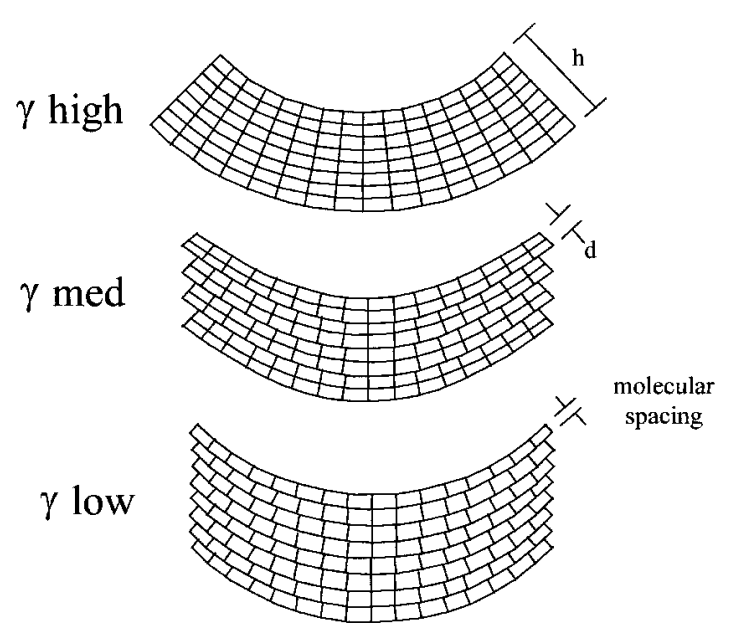

Figure 9. Transition between continuous, coherent and localized slip depending on the stacking fault energy

unrelaxed energy cost per unit area $W_{\mathrm{u}}$ of

$$
W_{\mathrm{u}}=E \kappa^{2} h^{3} /\left[24\left(1-\nu^{2}\right)\right]
$$

where $E$ is Young's modulus and $\nu$ is the Poisson ratio. If the curvature can be relaxed by interlayer sliding at a molecular length $a$, this energy is reduced:

$$
W_{\mathrm{r}}=E \kappa^{2} h a^{2} /\left[24\left(1-\nu^{2}\right)\right]
$$

Equation (4) corresponds to the energy required to bend each layer independently. Since $a \ll h$, this is a significant reduction, $\sim(a / h)^{2}$. These two limits correspond to the cases where the energy of intermolecular slip is either infinite or zero. To see what would happen in intermediate cases, consider now a model in which an energy per unit area $\gamma$ is required to introduce slip between layers. This would correspond to the stacking fault energy for this system. Then, for a film of total thickness $h$ sliding in units of some smaller size $d$, there would be $(h / d-1)$ new surfaces created, each of them with extra energy $\gamma$. In this case, the total energy would given by a suitably modified version of Eqn. (4), along with an extra term corresponding to the energy of the new surfaces:

$$
W_{\mathrm{d}}=E \kappa^{2} d^{2} h /\left[24\left(1-\nu^{2}\right)\right]+\gamma(h / d-1)
$$

Equations ((3)-(5)) predict that we should expect three regimes of behavior for curved crystals, depending of the value of the stacking fault energy $\gamma$ relative to the modulus $E$. For large $\gamma$, interlayer slip is costly and the system will bend as a coherent whole [Eqn. (3)]. For small $\gamma$, the system will slip independently on intermolecular layers of molecular spacing $a$ [Eqn. (4)]. For intermediate values of $\gamma$, the system should bend in domains of width $d$. The value of the stacking fault 


\section{PNBO HREM}

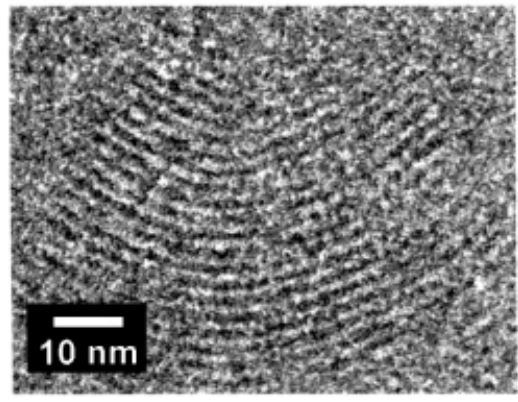

PNBT HREM

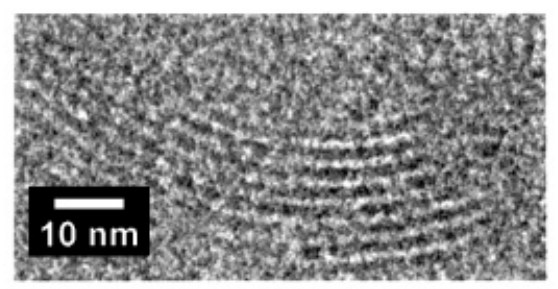

PNBO Curvature Map

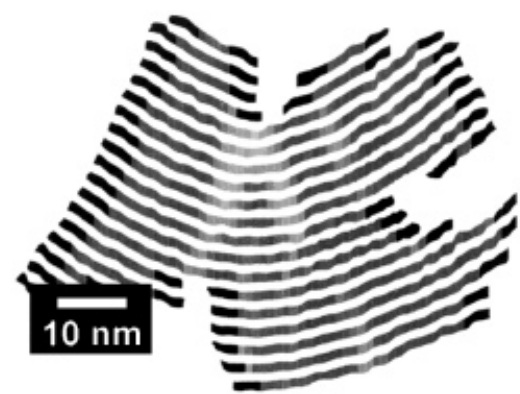

PNBT Curvature Map

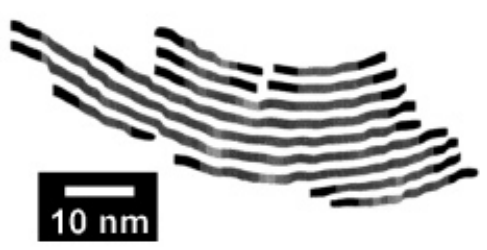

PNBO Curvature Histogram

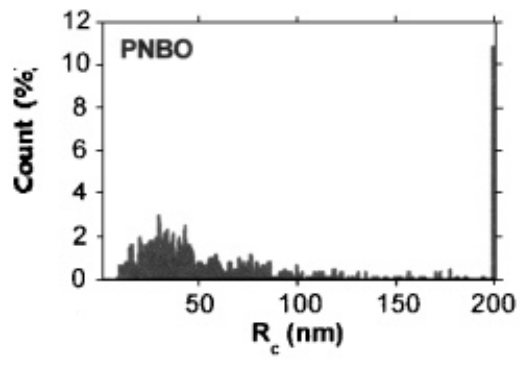

PNBT Curvature Histogram

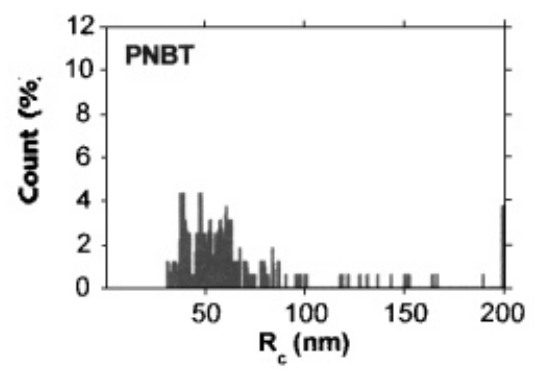

Figure 10. HREM images of PNBO and PNBT crystallites that exhibit different types of bending. The curvature maps and histograms show that PNBO exhibits localized bending, whereas the bending in PNBT is almost uniform throughout the crystallite. Reprinted from González-Ronda L, 'Microstructure and Defects of Electrooptically Active Bithiazoles and Bisoxazoles,' PhD Thesis, University of Michigan, 2000

spacing $d$ that minimizes the total energy $W_{\mathrm{d}}$ is given by taking the first derivative of Eqn. (5) and setting the result equal to zero:

$$
d=\left[12 \gamma\left(1-\nu^{2}\right) /\left(E \kappa^{2}\right)\right]^{1 / 3}
$$

The expected behavior is depicted schematically in Fig. 9. The stacking fault separation distance d should decrease with decreasing stacking fault energy $\gamma$, increasing modulus $E$ and increasing curvature $\kappa$. All of these assertions seem to be physically reasonable. This analysis may explain what factors control the transition from localized to continuous slip under different deformation conditions. Similar changes in the mechanisms of crystal plasticity have been observed in thin films of semicrystalline polymers, ${ }^{86,87}$ but the physical origins of these effects have not yet been well elucidated. From this analysis, localized slip should occur for high stacking fault energies, low modulus and at low curvatures. Conversely, we would expect molecular controlled continuous slip (small $d$ ) when the stacking fault energy $\gamma$ is low, the modulus $E$ high, and at high deformations $\kappa$.

$\mathrm{Wu}$, et al. have obtained experimental evidence for the bending of individual polymer molecules. ${ }^{88}$ They reported the direct HREM imaging of shape-persistent tetra(methoxy)tetra(octoxy)phthalocyaninatopoly(siloxane) (PcPS). The molecules were found to be locally stiff and fairly straight, leading to an estimate of 500-1000 nm for the persistence length. Sheiko et al. reported the direct imaging of polystyrene substituted poly(methyl methacrylate) molecular brushes by phase-contrast scanned probe microscopy. ${ }^{89}$ Evidence for local molecular bending was observed in the thin films, resulting in some cases in tight 'hairpins' observed in individual polymer chains.

We have established that the detailed mechanisms of
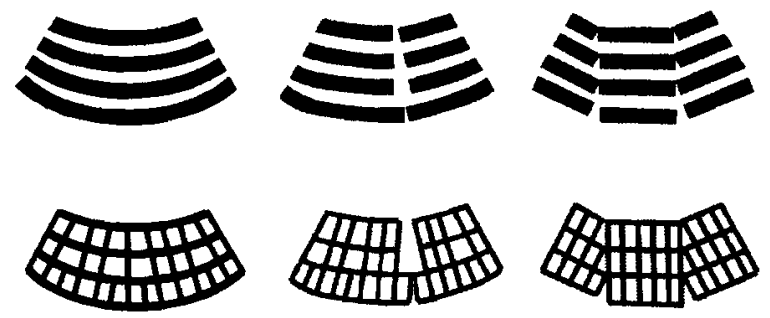

Figure 11. Schematic representation of continuous and localized bending and grain boundary-mediated bending. Reprinted from González-Ronda L, 'Microstructure and Defects of Electrooptically Active Bithiazoles and Bisoxazoles,' PhD Thesis, University of Michigan, 2000 
a)

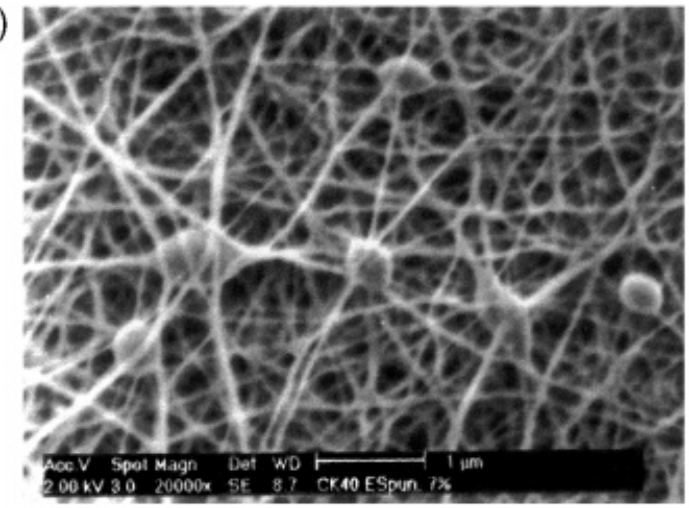

b)

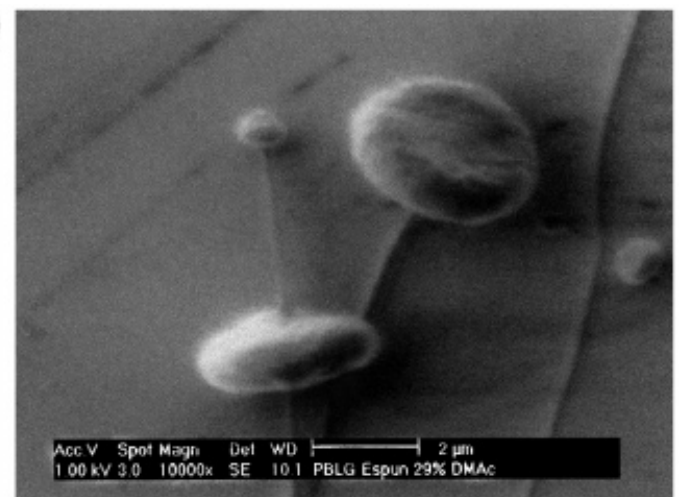

c)

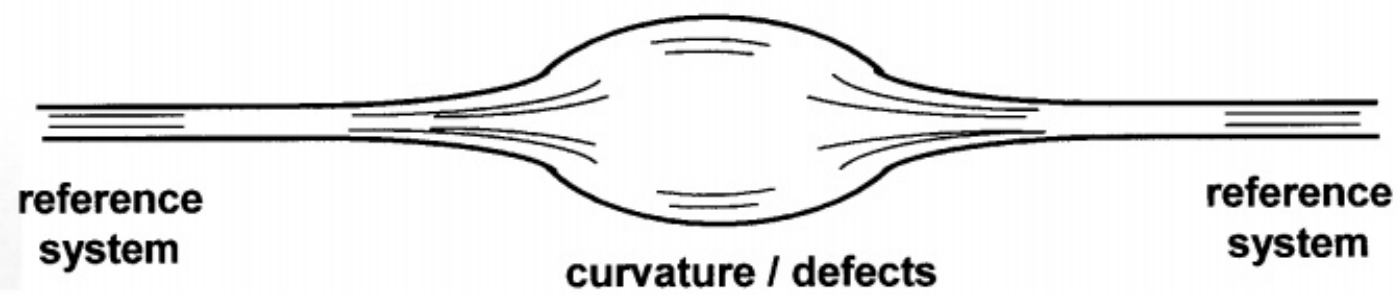

Figure 12. (a) Beaded electrospun nanofibers from an isotropic MPDI solution. (b) Discs on a string observed by electrospinning of a lyotropic PBLG solution. (c) Schematic representation of defect structure in beaded fibers

lattice curvature in crystallites are clearly system specific. ${ }^{14}$ HREM studies of poly(nonylbithiazole) (PNBT) and poly(nonylbisoxazole) (PNBO) thin films have shown lattice curvature with radii of the order of $50 \mathrm{~nm}$ along the polymer backbone (Fig. 10). Despite having almost identical chemical structures, the manner in which these molecules accommodate the deformation is fundamentally different (Fig. 11). PNBT undergoes a continuous, coherent deformation corresponding to a low stacking fault energy, which is presumably mediated by a uniform array of [001] dislocations. PNBO, on the other hand, exhibits highly localized bending corresponding to grain boundaries that accommodate the distortion by discontinuous deformation.

Molecular modeling of cyclic oligomers has indicated some differences in the persistence length between PNBT and PNBO. ${ }^{14}$ In addition to molecular stiffness, factors such as sample preparation, annealing and molecular weight are expected to also influence the manner in which polymers accommodate lattice bending. Differences in the crystallization condition during sample preparation may promote or hinder the development of grain boundaries, which are expected to lower the energy cost of bending or high stacking fault energies. ${ }^{85,90}$ The creation of grain boundaries might also be eased by chain-end segregation, which in turn is influenced by the chain-end density, i.e. the molecular weight of the sample.

A more detailed analysis of bending in polymer crystallites would require high-resolution imaging that allows the resolution of both the lattice planes which are themselves curving, and also the orthogonally oriented planes that correspond to the development of lateral order. Furthermore, a detailed analysis of the curvature dependence of localization of bending and slip relies on sample preparation techniques to control the morphology and radius of curvature in polymeric materials. This might be possible by electric field-mediated fiber spinning (electrospinning) of polymer solutions resulting in nanoscale fibers. ${ }^{91,92}$ for isotropic polymer solutions typically straight nanofibers or beaded structures [Fig. 12(a)] are obtained depending on the polymer concentration $^{91,92}$ and electrical field variations. ${ }^{93}$ Recently, we also observed a variety of more complex defect geometries by electrospinning of poly $(\gamma$-benzyl-L-glutamate) (PBLG) solutions from lyotropic nematic phase [Fig. 12(b)]. LD-HREM imaging of beaded fibers is expected to allow a detailed study of surface induced distortions of the polymer structure at high curvature [Fig. 12(c)].

Quantitative measurements of the curvature of a set of lattice fringes in high-resolution images is possible using an NIH Image macro developed by us (Fig. 10). ${ }^{14}$ Egolf et al. used a numerical approach to map the orientation and magnitude of the director fields in Rayleigh-Bernard cells and TEM images of microphase-separated block copolymers, ${ }^{94}$ which should be possible to adapt to highresolution TEM images. For a locally periodic pattern that can be approximated by a cosine function [A $=\cos (k x)$, where $x$ is a two-dimensional vector], the 


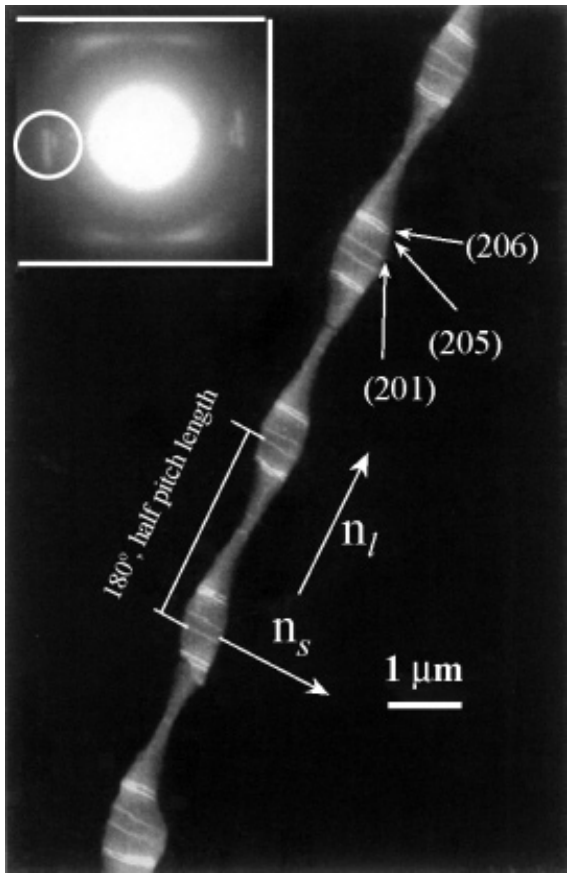

Figure 13. TEM dark-field image of the $\operatorname{PET}\left(R^{\star}\right)-9$ helical lamellar crystal from the (201), (205) and (206) diffraction arcs which are circled in the inset ED pattern. The bright bands on the crystal correspond to these three reflections. Reprinted from Li CY, Cheng S ZD, Ge JJ, Bai F, Zhang JZ, Mann IK, Harris FW, Chien L-C, Lotz B, 'Double-twist in Helical Lamellar Crystals in a Synthetic Main-chain Chiral Polyester Similar to Biological Polymers' Phys. Rev. Lett. 1999; 83: 524, with permission a)

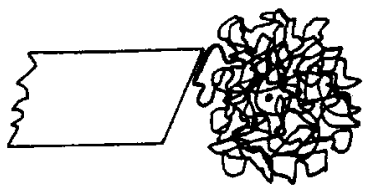

b)

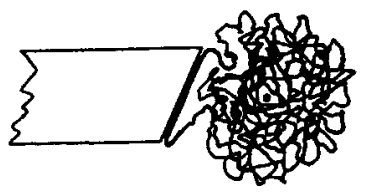

c)

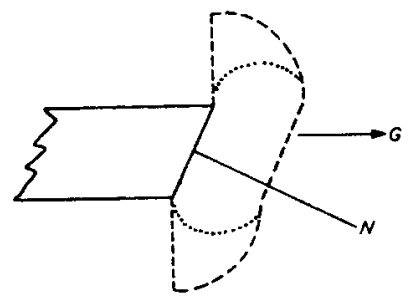

Figure 14. Representation of an oblique growth face encountering a coiled molecule in the melt. (a) Beginning of attachment. (b) Formation of the lower fold surface of relatively tight folding. (c) The dashed curve defines the region within which the center of mass of a molecule must lie in order that it may begin to crystallize. Reprinted from Keith $\mathrm{HD}$, Padden FJ Jr, 'Twisting Orientation and the Role of Transient State in Polymer Crystallization,' Polymer 1984; 25: 28, with permission from Elsevier Science

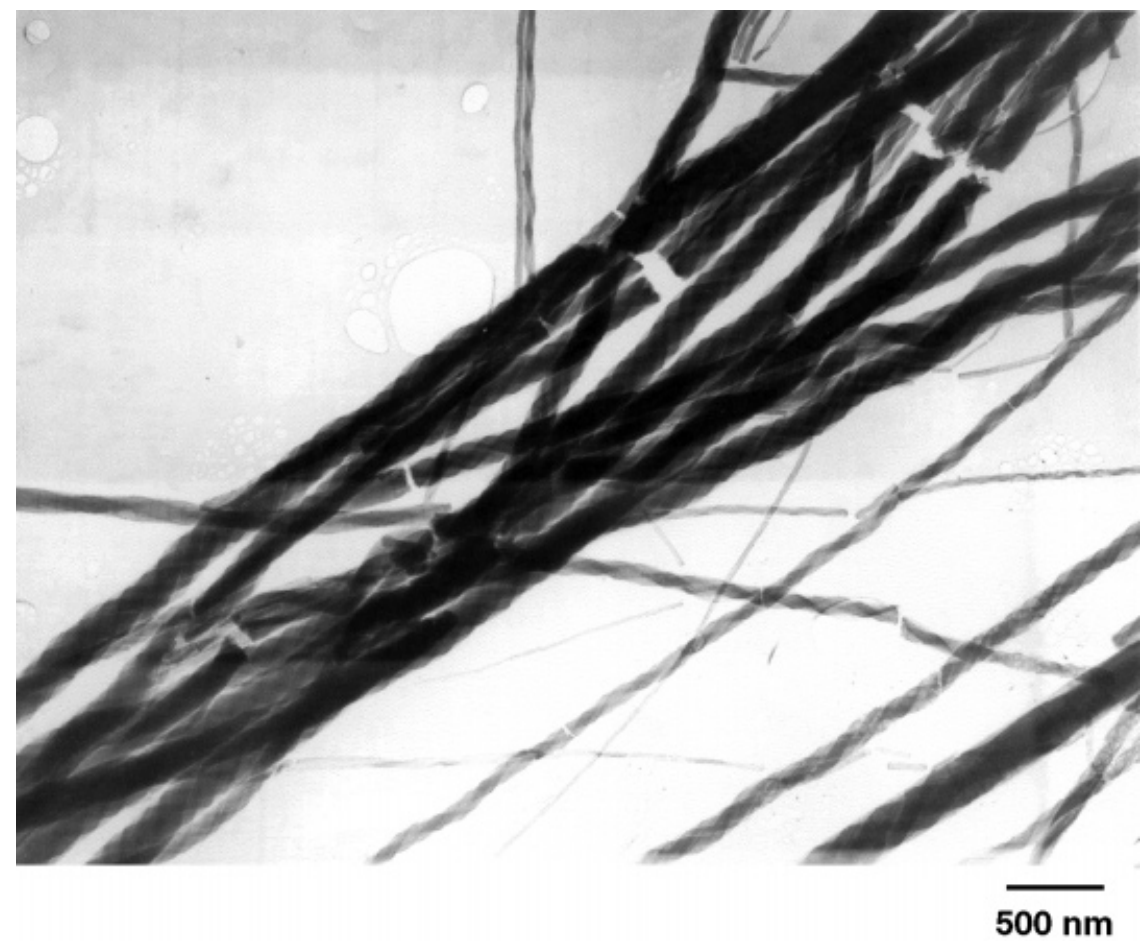

Figure 15. TEM image of uniform twisted MPDI fibers consisting of 1-8 individual strands. Reprinted from Lawrence DP, Jiang T, Martin DC, 'Twisted Crystals of Poly(meta-phenylene diisophthalamide) (Nomex $\left.{ }^{\mathbb{R}}\right)$;' Polymer Preprints 1997; 346, with permission 


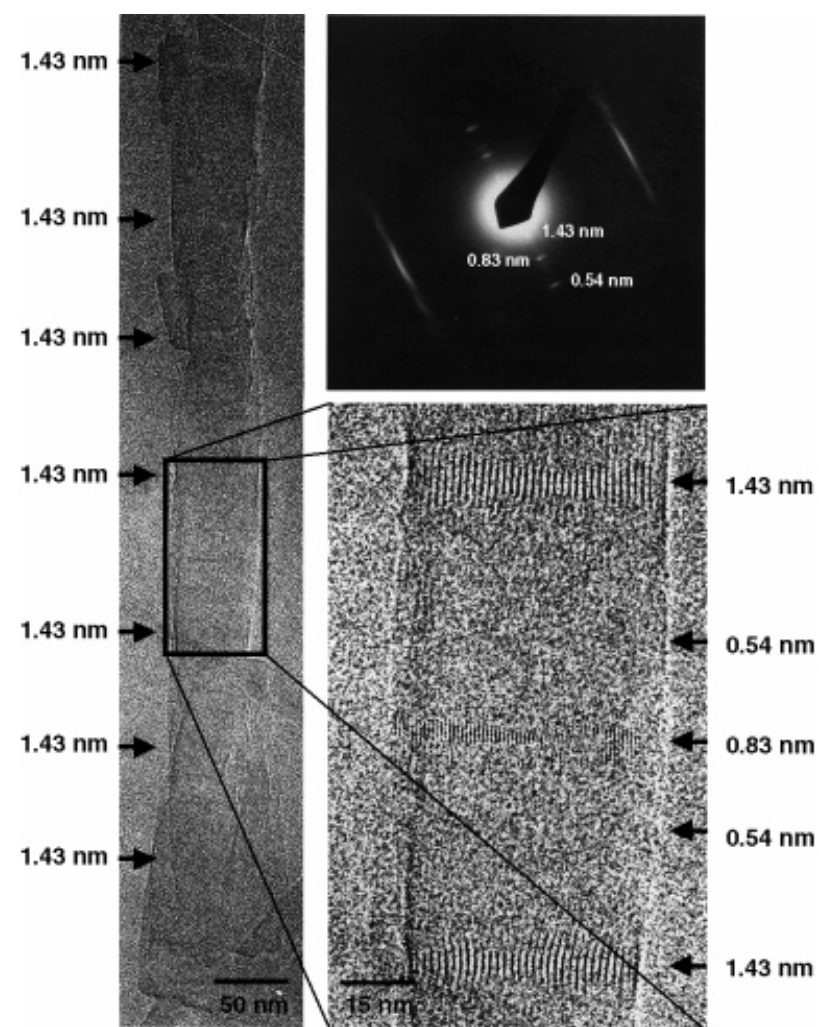

Figure 16. LD-HREM image and SAED pattern of a twisted MPDI fiber. The periodic appearance of three different sets of lattice fringes that run across the fiber indicate that each individual strand shows a continuous and uniform twist around its long axis

gradient of the phase of the wave $(k=\theta)$ defines a wavefield which characterizes the lattice orientation and spacing. This wavefield is singular at the core of a dislocation or a disclination. In analogy with the characteristic distortions of liquid crystals, ${ }^{95}$ bending of the lattice planes corresponds to splay of their normals, which is itself determined by the divergence of $k(k)$. Another potential method of interest was described by Bowman et al. in which local periodicities in images were extracted using wavelet transforms. ${ }^{96}$ Either of these algorithms should make it possible to map the local orientation of the lattice as a function of position within an individual crystallite and around defects.

\section{TWISTING IN POLYMER CRYSTALS}

A number of previous studies have shown either indirect or direct evidence for twisting in crystalline polymers. Geil and co-workers found that poly(benzyl glutamate) (PBLG) formed convoluted strands, large helical fibers, loops, or toroids depending on the solvent, precipitation temperature and concentration. ${ }^{15,97}$ Initially Blais and Geil reported that they observed both handedness for helical PBLG fibers, ${ }^{97}$ but later Rybnikar and Geil only observed one twist sense. ${ }^{15}$ It is still an ongoing discussion if and how chirality of the polymer chain is transmitted to higher order organizational levels. Saracovan et al. reported that they did not observe any morphological differences for solution-grown foldedchain single-crystal lamellae of poly(epichlorohydrin) or poly(propylene oxide) that could be related to chirality. ${ }^{98}$ On the other hand, Singfield et al. suggested that the chirality of the polymer main chain may determine the handedness of lamellar twisting and possibly the organization of lamellae in banded spherulites for meltcrystallized banded spherulites of poly(epichlorohydrin). ${ }^{99}$

$\mathrm{Li}$ and co-workers recently reported on twisted helical lamellar crystals of the synthetic chiral polyester $\operatorname{PET}\left(\mathrm{R}^{*}\right)-9$, where they observed only one twist sense for the lamellae. ${ }^{16,17}$ They used dark-field imaging to elucidate the structure of the helical lamellae and showed that the twisting results in a periodic change of the crystallographic orientation of the polymer chains along the helix axis (Fig. 13). Furthermore, they obtained evidence indicating that a lamella is also bent along its short crystal axis, giving rise to a so-called doubletwisted structure for the lamella.

Chirality is not a necessary prerequisite for the formation of twisted structures as twisted lamellae and banded spherulites have been observed for melt-crystallized achiral polymers such as PE and $\alpha-\mathrm{PVF}_{2} .{ }^{100}$ Keith and Padden explained the formation of twisted lamellae during melt-crystallization of achiral polymers using chain tilt (the molecular stems in chain folded crystals not being codirectional with the lamellar normal). ${ }^{100,101}$ The chain tilt results in tighter chain folds or different reentrant angles on one side of the crystal for a random coil polymer that is deposited from the center of the lamellae (Fig. 14). With new polymer coils approaching on average from the middle, this results in a higher surface stress on one side of the lamellae, which is released by twisting (for a more detailed explanation, see Keith and Padden ${ }^{101,}$ Appendix 1).

A complex and strongly twisted structure has recently been reported in our laboratory for the achiral $\operatorname{poly}\left(\mathrm{m}^{-}\right.$ phenylene diisopthalamide) (MPDI). ${ }^{102}$ TEM bright-field images exhibit regular twisted fibers of both handedness consisting of 1-8 individual strands wrapped around each other with intimate contact between the strands (Fig. 15). LD-HREM images indicate that the fibers exhibit yet another hierarchy of twisting. The periodic appearance of different sets of lattice fringes that run across the fiber indicates 3 that each individual strand shows a continuous and uniform twist around its long axis with a pitch of 150-800 nm (Fig. 16). ${ }^{102}$ Superimposed is the twist of the individual strands around each other visible in bright images with a pitch of $300-1000 \mathrm{~nm} .{ }^{102}$

In contrast to twisted lamellae crystals, our data indicates that the polymer chains adopt a helical molecular shape and are oriented nominally parallel to 
the macroscopic twist axis of the MPDI fiber. Streaking on layer lines in the diffraction pattern indicates that the twist distortions are locally mediated by the formation of lateral disorder resulting in a structure similar to a columnar liquid crystal (C. Kübel, D. P. Lawrence and D. C. Martin, presentation at the American Physical Society Meeting, Minneapolis, 2000, to be published). A more detailed analysis of the twisted crystals is expected to result in a better fundamental understanding of solid-state structures of polymers, dimensionality of order and the relationship between inter- and intramolecular interactions.

\section{Acknowledgements}

C.K. and D.C.M. thank the Alexander-von-Humboldt Foundation for fellowships. L.G.-R. thanks the Rackham Graduate School for a fellowship. L.F.D. thanks the NSF IGERT Center on Molecularly Designed Electronic, Photonic, and Nanostructured Materials. Support for this research was also provided by the National Science Foundation through grants DMR-9707974, DMR9704175, and DMR-0084304.

\section{REFERENCES}

1. Hunter SC, Nabarro FRN. Proc. R. Soc. London, Ser. A 1953; 220: 542

2. Queisser HJ, Haller EE. Science 1998; 281: 945.

3. Nakamura S. Science 1998; 281: 956.

4. Hellemans A. Science 1998, 281: 943.

5. Frank FC, Keller A, O’Conner A. Philos Mag. 1958; 3: 64

6. Keith HD, Passaglia E. J. Res. Natl. Bur. Stand. 1964; 68(A): 513.

7. Martin DC, Thomas EL. Mater. Res. Soc. Bull. 1987; 12(8): 27.

8. Martin DC, Thomas EL. Philos. Mag. A 1991; 64: 903.

9. Martin DC. Trends Polym. Sci. 1993; 1: 178.

10. Lovinger AJ, Katz HE, Dodabalapur A. Chem. Mater. 1998; 10: 3275.

11. Wilson PM, Martin DC. J. Mater. Res. 1992; 7: 3150.

12. Martin DC, Berger LL, Gardner KH. Macromolecules 1991; 24: 3921.

13. González-Ronda L, Martin DC. Macromolecules 1997; 30: 1524.

14. González-Ronda L. PhD Thesis, University of Michigan, 2000.

15. Rybnikar F, Geil PH. Biopolymers 1972; 11: 271.

16. Li CY, Yan D, Cheng SZD, Bai F, He T, Chien L-C, Harris FW, Lotz B. Macromolecules 1999; 32: 524.

17. Li CY, Cheng SZD, Ge JJ, Bai F, Zhang JZ, Mann IK, Harris FW, Chien L-C, Lotz B. Phys. Rev. Lett. 1999; 83: 4558.

18. Matare HF. Defect Electronics in Semiconductors. WileyInterscience: New York, 1971.

19. Barghout JY, Lorimer GW, Pilkington R, Prangnell PB. Mater. Sci. Forum 1996; 217-222: 975.

20. Shevchenko SA. J. Exp. Theor. Phys. 1999; 88: 66.

21. Garnier F. Chem. Rev. 1998; 227: 253.

22. Bao Z, Lovinger AJ. Chem. Mater. 1999; 11: 2607.

23. Horowitz G, Hajlaoui R, Bourguiga R, Hajlaoui M. Synth. Met. 1999; 101: 401.

24. Fichou D, Horowitz G, Nishikitani Y, Roncali J, Garnier F. Synth. Met. 1989; 28: C729.

25. Horowitz G, Fichou D, Peng XZ, Garnier F. Synth. Met. 1991; 41: 1127.

26. Akimichi H, Waragai K, Hotta S, Kano H, Sakaki H. Appl. Phys. Lett. 1991; 58: 1500.

27. Katz H, Torsi L, Dodababapur A. Chem. Mater. 1995; 7: 2235.
28. Dimitrakopoulos CD, Purushothaman S, Kymissis J, Callegari A, Shaw JM. Science 1999; 283: 822.

29. Schön JH, Berg S, Kloc Ch, Batlogg B. Science 2000; 287: 1022

30. Bao Z, Feng Y, Dodabalapur A, Raju VR, Lovinger AJ. Chem. Mater. 1997; 9: 1299.

31. Zaukelies DA. J. Appl.Phys. 1962; 11: 2797

32. Holland VF, Lindenmeyer PF. J. Appl. Phys. 1965; 36: 3049.

33. Predecki P, Statton WO. J. Appl. Phys. 1966; 37: 4053.

34. Shadrake LG, Guiu F. Philos. Mag. 1976; 34: 565.

35. Young RJ. Philos. Mag. 1974; 30: 85.

36. Young RJ. Materi. Forum 1988; 11: 210.

37. Crist B. Polym. Commun. 1989; 30(3): 69.

38. Crist B, Fisher CJ, Howard PR. Macromolecules 1989; 22: 1709.

39. Brooks NL, Mukhtar M. Polymer 2000; 41: 1475.

40. Erickson RH. Polymer 1985; 26: 733.

41. Rogozinsky AK, Bazhenov SL. Polymer 1992; 33: 1391.

42. Schuppert AA. Makomol. Chem. Theor. Simul. 1993; 2: 643.

43. Wegner G. Z. Naturf. Corsch., Teil B 1969; 24 : 824.

44. Liao J, Martin DC. Macromolecules 1996; 29: 568.

45. Martin DC, Wilson PM, Liao J. Polym. Prepr. 1992; 33: 245.

46. Martin DC, Thomas EL. Polymer 1995; 36: 1743.

47. Tsuji M, Kohjiya S. Prog. Polym. Sci. 1995; 20: 259.

48. Voigt-Martin IG. Acta Polym. 1996; 47: 311.

49. Plummer CJG, Gensler, R, Kausch HH. Colloid Polym. Sci. 1997; 275: 1068

50. Hudson SD. Curr. Opin. Colloid Interface Sci. 1998; 3: 125.

51. Mazerolles L, Folch S, Colomban P. Macromolecules 1999; 32 8504.

52. Loiacono MJ, Granstrom EL, Frisbie CD. J. Phys. Chem. B 1998; 102: 1679

53. Kelley TW, Granstrom EL, Frisbie CD. Adv. Mater. 1999; 11: 261.

54. Kelley TM, Frisbie CD. J. Vac. Sci. Technol. B in press.

55. Sutton AP, Balluffi RW. Acta Metall. Mater. 1987; 35: 2177.

56. Sutton AP, Balluffi RW. Interfaces in Crystalline Materials. Oxford Science Publications: Oxford, 1995.

57. Wilson PM, Martin DC. Macromolecules 1996; 29: 842.

58. Chaikin PM, Lubensky TC. Cambridge University Press: Cambridge, 1995.

59. Weertman, Weertman. Elementary Dislocation Theory. Macmillan: New York, 1964.

60. Steeds JW. Anisotropic Elasticity Theory of Dislocations. Clarendon Press: Oxford, 1973.

61. Choi HC, Schwartzmann AF, Kim K-S. Mater. Res. Soc. Symp. Proc. 1992; 239: 419.

62. Hull D, Bacon DJ. Introduction to Dislocations (3rd edn), International Series on Materials Science and Technology, vol. 37. Butterworth-Heineman: Oxford, 1984.

63. Hirth JP, Lothe J. Theory of Dislocations (2nd edn). WileyInterscience: New York, 1982.

64. Rodriguez F. Principles of Polymer Systems. Hemisphere: Washington, DC, 1982.

65. Illers K-H, Hendus H. Makromol. Chem. 1968; 113: 1

66. Mokichev NN, Pakhomov LG. Sov. Phys. Solid State 1982; 24 1925.

67. Bacon DJ, Tharmalingam K. J. Mater. Sci. 1983; 18: 884

68. Ide N, Okada I, Kojima K. J. Phys.: Condens. Matter 1990; 2: 5489 .

69. Ide N, Okada I, Kojima K. J. Phys.: Condens. Matter 1993; 5: 3151.

70. Kuklja MM, Kunz AB. J. Appl. Phys. 2000; 87: 2215.

71. Zwanger MS, Banhart F, Seeger A. J. Cryst. Growth 1996; 163 445.

72. Banhart F, Ajayan PM. Adv. Mater. 1997; 9: 261.

73. Putnis A. Introduction to Mineral Sciences. Cambridge University Press: Cambridge, 1992.

74. Ozin GA, Yang H, Sokolov I, Coombs M. Adv. Mater. 1997; 9: 662.

75. Yang H, Ozin GA, Kresge CT. Adv. Mater. 1998; 10: 883.

76. Martin DC, Thomas EL. J. Mater. Sci. 1991; 26: 5171.

77. Jones M-CG, Martin DC. J. Mater. Sci. 1997; 32: 2291.

78. Wilson PM PhD Dissertation, University of Michigan, 1994

79. Katagi H, Kasai H, Okada S, Oikawa H, Matsuda H, Nakanishi H. Pure Appl. Chem. 1997; A34: 2013. 
80. Samori P, Francke V, Müllen K, Rabe JP. Thin Solid Films 1998; 336: 13.

81. Samori P, Sikharulidze I, Francke V, Müllen K, Rabe JP. Nanotechnology 1999; 10: 77.

82. Nye JF. Acta Metall. 1953; 1: 153.

83. Read WT Jr. Acta Metall. 1957; 5: 83.

84. Bilby BA, Gardner LRT, Smith E. Acta Metall. 1958; 6: 29.

85. Srolovitz DJ, Safran SA, Tenne R. Phys. Rev. E 1994; 49: 5260.

86. Brady JM, Thomas EL. J. Mater. Sci. 1989; 24: 3311.

87. Brady JM, Thomas EL. J. Mater. Sci. 1989; 24: 3319.

88. Wu J, Lieser G, Wegner G. Adv. Mater. 1996; 8: 151.

89. Sheiko SS, Gerle M, Fischer K, Schmidt M, Möller M. Langmuir 1997; 13: 5368.

90. Nabarro FRN. Theory of Crystal Dislocations. Dover: New York, 1987.

91. Buchko CJ, Chen LC, Shen Y, Martin DC. Polymer 1999; 40: 7397.
92. Doshi J, Reneker DH. J. Electrostat. 1995; 35: 151.

93. Johnson MA PhD Thesis, University of Michigan, 1999.

94. Egolf DA, Melnikov IV, Bodenschatz E. Phys. Rev. Lett. 1998; 80: 3228.

95. De Gennes PG, Prost J. The Physics of Liquid Crystals (2nd edn). Clarendon Press: Oxford, 1993.

96. Bowman C, Passot T, Assenheimer M, Newell AC. Physica D 1998; 119: 250.

97. Blais JJBP, Geil PH. J. Ultrastruct. Res. 1968; 22: 303.

98. Saracovan I, Cox JK, Revol J-F, Manley RSJ, Brown GR. Macromolecules 1999; 32: 717.

99. Singfield KL, Klass JM, Brown GR. Macromolecules 1995; 28 : 8006.

100. Keith HD, Padden FJ Jr. Macromolecules 1996; 29: 7776.

101. Keith HD, Padden FJ Jr. Polymer 1984; 25: 28.

102. Lawrence DP, Jiang T, Martin DC. Polym. Prepr. 1997; 345. 